\title{
Longitudinal Assessment of Swimming Performance in the 200-m Freestyle Event
}

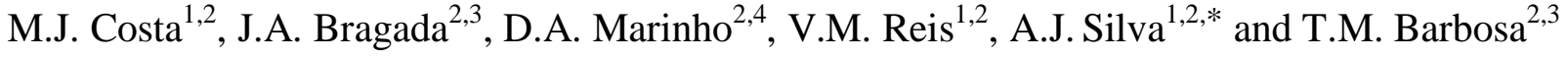 \\ ${ }^{I}$ Department of Sport Sciences, Univesity of Trás-os-Montes and Alto Douro, Vila Real, Portugal \\ ${ }^{2}$ Research Center in Sports Science, Health and Human Development, Vila Real, Portugal \\ ${ }^{3}$ Department of Sport Sciences, Polytechnic Institute of Bragança, Bragança, Portugal \\ ${ }^{4}$ Department of Sport Sciences, Univesity of Beira-Interior, Covilhã, Portugal
}

\begin{abstract}
The aim of this study was to track and analyze the 200-m Freestyle performance stability throughout elite swimmer's career. 29 Portuguese male top-50 were analyzed for seven consecutive seasons between 12 and 18 years old. Best performances were collected from ranking tables. Longitudinal assessment was performed based on two approaches: (i) mean stability was analyzed by descriptive statistics and ANOVA repeated measures for each season followed by a post-hoc test (Bonferroni test), (ii) normative stability was analyzed with self-correlation (Malina, 2001) and the Cohen's Kappa tracking index (Landis and Koch, 1977). There was a 200-m Freestyle performance enhancement from children to adult age. The overall career performance prediction was moderate. The change from 13 to 14 years can be a milestone, where the ability to predict the final swimmer's performance level strongly increases.
\end{abstract}

Keywords: stability, prediction, tracking, performance, elite swimmers

\section{INTRODUCTION}

Several studies have been carried out in order to identify the factors that can predict swimming performance. However, all these studies only analyzed determinant factors in a single time moment. It was not taken into account the longitudinal stability and change, as a result of individual development, new training methods and technological sophistication. Also was demonstrated that progression and variability of performance between competitions are useful for researchers and practitioners interested in factors that affect performance [1]. Indeed, the number of studies about swimming performance, adopting longitudinal approaches is reduced [e.g., 1, 2]. So far, appears not exist any research tracking the change and stability of swimmer's performance. The aim of this study was to track and analyze the stability of the 200-m freestyle performance throughout the swimmer's career, from children to adult age.

\section{METHODS}

\section{Subjects}

Twenty nine Portuguese male swimmers were analyzed for seven consecutive seasons between 12 and 18 years old.

\section{Data Collection}

Swimming performance was collected using the best personal time in the 200-m Freestyle event in each season.

*Address correspondence to this author at the Department of Sport Sciences, University of Trás-os-Montes and Alto Douro, Vila Real, Portugal;

Tel: +351259350000; E-mail: Ajsilva@utad.pt
Performances were collected from official competitions, on a short course pool (regional, national or international level). The official times were consulted on rankings tables provided by the Portuguese Swimming Federation and in an internet database (www.swimrankings.net, March 2009). At the age of 18,7 were international level swimmers, 15 national level ones and 7 regional level.

\section{Statistical Procedures}

An exploratory data analysis was performed to identify possible errors in data input, outliers and to assess data normality (Shapiro-Wilk test).

Longitudinal assessment was made based in two approaches: (i) mean stability; (ii) normative stability. For mean stability quartiles, means plus standard deviations were computed for each chronological age. Data variation was analyzed with ANOVA repeated measures followed by a post-hoc test (Bonferroni test). Normative stability was analyzed with self-correlation (Correlation of Pearson) between the performances throughout the seven seasons. It was defined that stability was: i) high if $r \geq 0.60$; (ii) moderate if $0.30 \leq r<0.60$; (iii) low if $r<0.30$, as suggested by Malina [3]. It was also calculated the Cohen's Kappa (K), with a confidence interval of $95 \%$. The qualitative interpretation of Kappa values was: (i) excellent if $\mathrm{K} \geq 0.75$; (ii) moderate if $0.40 \leq \mathrm{K}<0.75$; (iii) low if $\mathrm{K}<0.40$ [4] .

All statistical procedures were computed with SPSS software's (v. 13.0, Apache Software Foundation, Chicago, IL, USA). However, $K$ value was computed with the Longitudinal Data Analysis software (v. 3.2, Dallas, USA). Significant level was set at $\mathrm{p}<0.05$. 


\section{RESULTS AND DISCUSSION}

There was a trend for a performance improvement throughout the swimmer's career from children to adult age (Fig. 1). Significant variations in the mean swim performance were verified $[F(1,28)=11815.908 ; p<0.01]$. Posthoc test verified significant variations between all chronological ages analyzed $(\mathrm{p}<0.01)$ except for the pair wise comparison between 16-17 years old and 17-18 years old that were not-significant.

This can be explained by changes that occurred during swimmers development, in different maturational stages. As swimming performance is determined by different parameters, the swimmers development will affect the energy cost of swimming [5]. Changes in anthropometric characteristics, like body length and body mass, are identified as causes to increase energy cost [6]. Along with these morphological changes, development shows an increase in swimmers muscular strength. It appears that hormonal development that occurs during maturation has a determinant role in increasing muscle size [7]. Constant training also contributes to improve swimming technique to obtain gains in performance [8].
The self-correlation values (Table 1) ranged between moderate and high throughout the swimmer's career. Stability becomes high $(r=0.644)$ from 14 years old until 18 years old. However this increase reveals a "braking effect" in the improvement of swimmers performance starting at 16 years old. This may be associated with: (i) a maximal level of external training load reached by the swimmers, which is more difficult to overcome; (ii) a decrease in physiological functional capacity with age [9] or; (iii) a slowdown or stagnation in the development of anthropometric characteristics [10].

The $\mathrm{K}$ value, expressing the stability throughout the overall swimmer's career, was moderate $(K=0.455 \pm 0.057)$ with $0.398 \leq \mathrm{K} \leq 0.512$ for a $95 \%$ of confidence interval. Based on a so long career, it is clearly that swimmers performance stability is difficult to maintain always on a high level. However, the change from 14 to 15 years old appears to show a sharp increase in stability. This can be explained by: i) maturational process, that provides greater availability for training process, in order to obtain more ambitious performances, ii) consolidation of values and culture

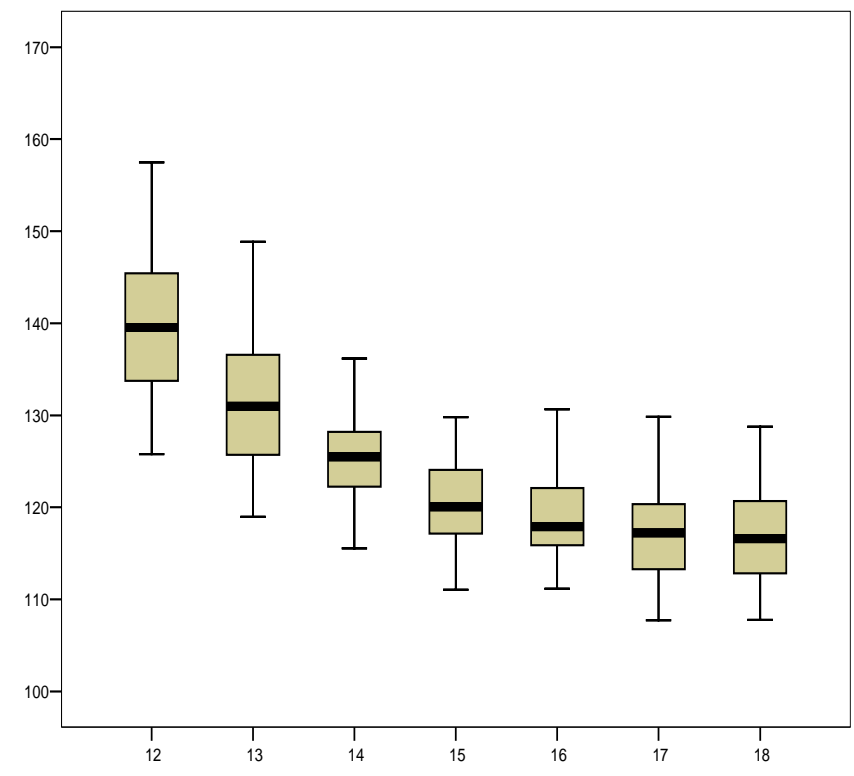

Fig. (1). Diagram of performance extremes and quartiles.

Table 1. Self-Correlation Values Throughout Swimmer's Career

\begin{tabular}{|c|c|c|c|c|c|c|c|}
\hline Chronological Age & $\mathbf{1 2}$ & $\mathbf{1 3}$ & $\mathbf{1 4}$ & $\mathbf{1 5}$ & $\mathbf{1 6}$ & $\mathbf{1 7}$ \\
\hline \hline 12 & 1 & & & & & \\
\hline 13 & $0.902^{*}$ & 1 & & & & \\
\hline 14 & $0.751^{*}$ & $0.882^{*}$ & 1 & & & \\
\hline 15 & $0.672^{*}$ & $0.748^{*}$ & $0.841^{*}$ & 1 & & \\
\hline 16 & $0.593^{*}$ & $0.699^{*}$ & $0.779^{*}$ & $0.929^{*}$ & 1 & \\
\hline 17 & $0.536^{*}$ & $0.589^{*}$ & $0.686^{*}$ & $0.850^{*}$ & $0.884^{*}$ & 1 \\
\hline 18 & $0.536^{*}$ & $0.561^{*}$ & $0.644^{*}$ & $0.813^{*}$ & $0.862^{*}$ & $0.955^{*}$ & 1 \\
\hline
\end{tabular}

$* \mathrm{p}<0.05$. 
of swimming performance acquired throughout swimmer's career.

As a conclusion, the prediction of adult swimmer's performance level, based on children performance is moderate. However, it seems that the change from 13 to 14 years old can be a milestone. At moment the ability to predict the swimmer's performance level increases strongly.

\section{ACKNOWLEDGEMENTS}

To the Portuguese Swimming Federation for providing the official times.

\section{REFERENCES}

Pyne D, Trewin C, Hopkins W. Progression and variability of competitive performance of Olympic swimmers. J Sports Sci 2004; 22(7): 613-20

[2] Stewart A, Hopkins W. Consistency of swimming performance within and between competitions. Med Sci Sports Exerc 2000; 32(5): 997-1001.
[3] Malina RM. Adherence to physical activity from childhood to adulthood: a perspective forma tracking studies. Quest 2001; 53: 346-55.

[4] Landis JR, Koch GG. The measurement of observer agreement for categorical data. Biometrics 1977; 37: 439-46.

[5] Kjendlie PL, Ingjer F, Madsen O, Stallman RK, Stray-Gundersen J. Differences in the energy cost between children and adults during front crawl swimming. Eur J Appl Physiol 2003; 91: 473-80.

[6] Chatard JC, Padilla S, Carzorla G, Lacour JR. Influence of body height, weight, hydrostatic lift and training on the energy cost of the front crawl. N Z L Sports Med 1985; 13: 82-4.

[7] Matos N, Winsley RL. Trainability of young athletes and overtraining. J Sport Sci 2007; 6: 353-67.

[8] Zamparo P. Effects of age and gender on the propelling efficiency of the arm stroke. Eur J Appl Physiol 2006; 97: 52-8.

[9] Donato A, Tench K, Glueck D, Seals D, Eskurza I, Tanaka H. Declines in physiological functional capacity with age: a longitudinal study in peak swimming performance. J Appl Physiol 2003; 94: 764-69.

[10] Malina RM, Bouchard C. Growth, maturation, and physical activity. In: Risk factors and children's health. Champaign, Illinois: Human Kinetics Books 1991.

(C) Costa et al.; Licensee Bentham Open.

This is an open access article licensed under the terms of the Creative Commons Attribution Non-Commercial License (http://creativecommons.org/licenses/by-nc/3.0/) which permits unrestricted, non-commercial use, distribution and reproduction in any medium, provided the work is properly cited. 